nations and preparing for boosters to optimize protection makes sense. But currently, the vaccine supply is limited and this is a zero-sum game.

In fact, it has been a zero-sum game for some time, and will continue to be until supply is dramatically increased via the TRIPS (Trade-Related Aspects of Intellectual Property Rights) waiver and technology transfers. Since rich nations have procured a giant share of current and future doses, booster vaccination programs will mean that low-income countries will have to wait longer to get their orders fulfilled. Pharmaceutical companies will typically give priority to rich nations that are likely to pay premium prices. Booster doses in rich countries is a lucrative market.

Rich countries claimed they could both vaccinate their adolescents in early May while still contributing to global vaccine campaigns, but the reality of the situation was different. From May to August 2021, high-income countries had given a first dose of vaccine to $28 \%$ of their population, while low-income countries had given a mere $0.7 \%$ of their population their first dose (Fig. 1).

The population in high-income countries is nearly 50 times more vaccinated than that in low-income countries. Many lowand middle-income countries are awaiting contracts from manufacturers and COVAX (the vaccines pillar of the Access to COVID19 Tools (ACT) Accelerator) to be filled while dealing with a Delta wave, while other countries have more vaccine than eligible residents. In fact, several countries, including the United States, Canada, Israel and the United Kingdom, are now throwing away expired or expiring vaccines.

Given the global community in this vaccine and scientific era, it is unconscionable for the outcomes of COVID-19 to be determined simply by the country one resides in. Rich nations that have vaccinated most of their population should consider reallocation of excess vaccinations, while also being mindful of the effect of future purchases on lower-income countries awaiting orders to be fulfilled. We urge everyone, wherever they live, to become outspoken advocates for vaccine equity $^{10}$. Our collective future is at stake.

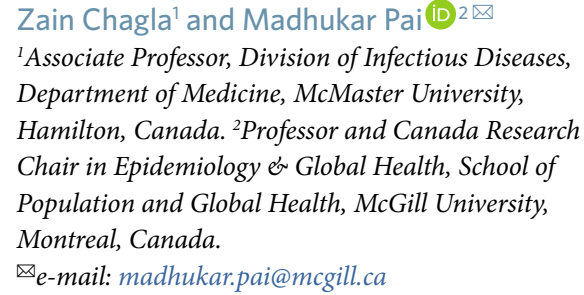

Published online: 31 August 2021

https://doi.org/10.1038/s41591-021-01494-4

References

1. Thomas, S. J. et al. Preprint at medRxiv https://doi. org/10.1101/2021.07.28.21261159 (2021)

2. Moderna. https://investors.modernatx.com/news-releases/ news-release-details/moderna-reports-second-quarter-fiscal-year2021-financial (5 August 2021).

3. Public Health England. https://assets.publishing.service.gov uk/government/uploads/system/uploads/attachment_data/ file/1005517/Technical_Briefing 19.pdf (23 July 2021).

4. Lopez Bernal, J. et al. N. Engl. J. Med. https://doi.org/10.1056/ NEJMoa2108891 (2021).

5. Nasreen, S. et al. Preprint at medRxiv https://doi. org/10.1101/2021.06.28.21259420 (2021).

6. Kamar, N. et al. N. Engl. J. Med. https://doi.org/10.1056/ NEJMc2108861 (2021).

7. Maxmen, A. Nature https://doi.org/10.1038/d41586-021-02109-1 (2021).

8. Anand, A., Sandefur, J. \& Subramanian, A. Center for Global Development https://www.cgdev.org/sites/default/files/threenew-estimates-indias-all-cause-excess-mortality-duringcovid-19-pandemic.pdf (July 2021).

9. World Health Organization. https://www.who.int/news/item/1507-2021-covid-19-pandemic-leads-to-major-backsliding-on-child hood-vaccinations-new-who-unicef-data-shows (15 July 2021).

10. Pai, M. Nature Portfolio https://naturemicrobiologycommunity. nature.com/posts/10-reasons-why-everyo ne-should-advocate-for-covid-19-vaccine-equity?channel_id=25 49-coronaviruses-past-present-and-future (15 April 2021).

\section{Author contributions}

Both authors were involved with the conceptualization, writing, and review of this Correspondence.

\title{
COVID-19 vaccination gives hope to eradicate polio
}

To the Editor-Before the emergence of COVID-19, polio was considered to be one of the most challenging infectious diseases of international concern, despite the consistent efforts of the Global Polio Eradication Initiative (GPEI) program to vaccinate every child in the endemic countries, Pakistan and Afghanistan. In June 2021, the GPEI launched a revised and strengthened plan 'Polio Eradication Strategy 2022-2026: Delivering on a Promise', replacing the previous plan from 2019 to 2023 , to overcome the continuing challenges in polio eradication ${ }^{1}$.

In the past five years, $67 \%$ of the total wild poliovirus type 1 (WPV1) cases were reported from Pakistan, with the remaining 33\% from Afghanistan, and all other cases worldwide were due to circulatory vaccine-derived poliovirus ${ }^{2}$. Unlike routine pediatric vaccination, polio vaccination campaigns have been particularly hampered by conspiracy theories and poor awareness about the lifesaving benefits of vaccines, at least among some parents ${ }^{3}$. Consequently, lack of vaccination and incomplete vaccination have been the main factors for the emergence of WPV1 and circulatory vaccine-derived poliovirus (cVDPV2) cases in both countries. However, the recent global vaccination drive against COVID-19 has raised public understanding of the need for vaccines in general, including polio vaccine. Although COVID-19 and polio have distinct physiological manifestations, both diseases share the commonality of vaccination as the only preventive measure.

The COVID-19 pandemic is continuously playing havoc with 211.7 million cases and 4.43 million deaths (as of 23 August 2021) ${ }^{4}$. Although the emergence of different variants of SARS-CoV-2, such as the Delta variant, has challenged control strategies, the WHO-approved vaccines have diminished the drastic situation in terms of mortality and morbidity. As of 23 August 2021, 24.6\% of the world population had been fully vaccinated, $32.7 \%$ had received at least a single dose and 33.56 million doses are being administered on daily basis ${ }^{5}$.

However, the pandemic has compromised the fragile and oversaturated healthcare systems in low- and middle-income countries, including the routine pediatric immunization against vaccine-preventable diseases. For example, inadequate vaccination against measles has listed Pakistan and Afghanistan among the top ten countries with the highest measles cases in $2021^{6}$. According to UNICEF, the temporary suspension of polio vaccination campaigning in both countries 


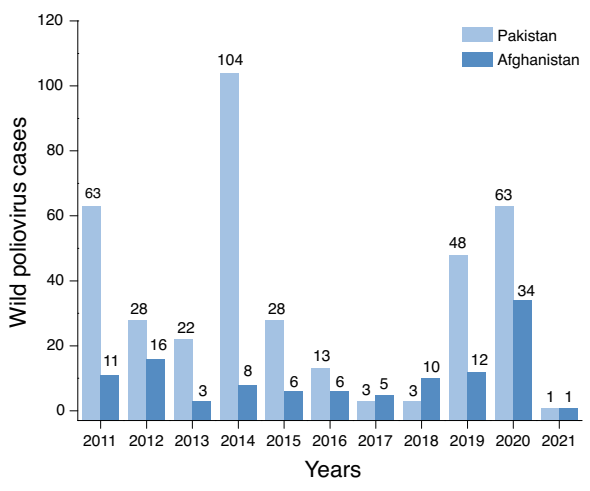

Fig. 1 | Wild poliovirus cases reported in the first eight months in Pakistan and Afghanistan. Data shown are for January to August, 2011-2021. Credit: Global Polio Eradication Initiative

in March 2020, to circumvent the potential spread of COVID-19, had left 50 million children unimmunized ${ }^{7}$. Although the polio vaccination campaign resumed in July 2020, the number of polio cases (WPV1 and cVDPV2) had reached 219 in Pakistan and 364 in Afghanistan by the end of $2020^{8}$, which accounted for $47.5 \%$ of all global polio cases last year.

Nevertheless, the emergence of 9 polio cases (1 WPV1, 8 cVDPV2) in Pakistan and 44 cases ( 1 WPV1, 43 cVDPV2) in Afghanistan between January and August $2021^{8}$ indicates a sharp decline, with most of the cases being vaccine-derived. With only two cases of WPV1 in both countries, 2021 is on track to have the lowest toll of polio virus in a decade (Fig. 1). This reduction in polio may be attributed to efficient governmental interventions and improved awareness of vaccination among the general public.

Amidst the fourth wave of COVID-19 and the potential threat of the Delta variant, Pakistan has prioritized polio vaccination campaigns and more than 40 million children have received two doses of the polio vaccine in $2021^{9}$. By contrast, Afghanistan is struggling to continue their polio vaccination campaigns ${ }^{10}$; in part due to instability and the changing political dynamics of the country.

Vaccine hesitancy in Pakistan and Afghanistan has been a considerable problem for polio eradication in the past. The widespread coverage and administration of COVID-19 vaccines around the world has increased awareness about the lifesaving effects of vaccines, and this has had an effect on public awareness in polio endemic countries. There remains a need for booster doses of polio vaccines, especially in the tropics. There is also a need to implement GPEI's “the switch" strategy to phase out the oral polio vaccine in routine immunizations, which may reduce vaccine-derived polio cases by more than 90\%. Nevertheless, COVID-19 vaccination centers remain an ideal setting to increase awareness of polio vaccines, to protect the lives of children, and finally move towards eradication.
Muhammad Rizwan', Murad Ali' and Bilal Haider Abbasi $^{2}$

${ }^{1}$ Centre for Biotechnology and Microbiology, University of Swat, Swat, Pakistan. ${ }^{2}$ Department of Biotechnology, Quaid-i-Azam University, Islamabad, Pakistan.

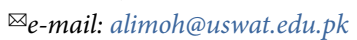

Published online: 4 October 2021

https://doi.org/10.1038/s41591-021-01518-Z

\section{References}

1. WHO. https://www.who.int/news/item/10-06-2021-countriesreaffirm-commitment-to-ending-polio-at-launch-of-neweradication-strategy (accessed 11 August 2021).

2. Global Polio Eradication Initiative. https://polioeradication.org/ polio-today/polio-now/wild-poliovirus-list (accessed 11 August 2021).

3. Ali, M. et al. Lancet 394, 915-916 (2019).

4. Worldometer. https://www.worldometers.info/coronavirus (accessed 11 August 2021).

5. Ourworldindata. https://ourworldindata.org/covid-vaccinations (accessed 11 August 2021)

6. Centers for Disease Control and Prevention. https://www.cdc. gov/globalhealth/measles/data/global-measles-outbreaks.html (accessed 11 August 2021)

7. UNICEF. https://www.unicef.org/press-releases/poliovaccination-campaigns-resume-afghanistan-and-pakistan-aftercovid-19 (accessed 11 August 2021).

8. Global Polio Eradication Initiative. https://polioeradication.org/ polio-today/polio-now/this-week (accessed 11 August 2021).

9. Reliefweb. https://reliefweb.int/report/pakistan/ more-40-million-children-received-vaccination-duringnationwide-anti-polio (accessed 11 August 2021).

10. WHO. http://www.emro.who.int/afg/afghanistan-news/ third-national-polio-immunization-campaign-beginsin-afghanistan.html (accessed 11 August 2021).

\section{Author contributions}

M.A. was responsible for the concept, literature search and writing; J.K. and B.H.A. revised the letter; N.A., H.K. and A.I. helped in data collection; M.R. and M.A. helped in data analysis, interpretation and writing.

\section{Health-based civic engagement is a professional responsibility}

To the Editor - Since the 2020 US presidential election, there has been a nationwide assault on voting rights, with 18 states enacting 30 new laws that restrict voting access, and hundreds of similar bills marching through state legislatures ${ }^{1}$. Recent restrictions draw comparisons with the literacy tests and poll taxes of the Jim Crow era. Indeed, much of this voter suppression is targeted toward Black people and Indigenous Americans and people of color, all of whom have long borne the violent burden of institutionalized racism and are survivors of extensive, punitive disparities in all areas of life. Health professionals must champion patients' right to vote to protect health and deracinate inequitable medical practices, building on the efforts of organizations such as VotER and VoteHealth 2020.

The interdependency between voting and health, as well as the power of policy to address social determinants of health, underscore why civic-health promotion must become a professional responsibility for healthcare workers. From registering to vote to attending neighborhood town halls to engaging government representatives, civic health refers to the ability of a community to come together and address public concerns. Civic health is a powerful indicator of overall health, with lower voting rates consistently associated with poorer health; for instance, patients with chronic illnesses are less likely to vote than healthy individuals ${ }^{2}$. Civic and health engagement amplify and reciprocate one another, with longitudinal studies demonstrating bidirectional causal pathways. 